\title{
I media del servizio pubblico in Europa: un silenzioso mutamento di modelli?
}

\author{
Katharine Sarikakis, Università di Vienna ${ }^{1}$
}

Il seguente dibattito affronterà la questione degli spazi di comunicazione pubblici intesi come spazi per la partecipazione dei cittadini al pubblico dibattito e, in definitiva, alle politiche pubbliche.

Per una democrazia che funzioni, l'esistenza di istituzioni che si adoperino a servizio del bene pubblico è (o dovrebbe essere) essenziale. I concetti di "bene pubblico" e di "pubblico interesse" sono inestricabilmente collegati al perseguimento della "buona vita" come esigenza politica e obiettivo delle politiche pubbliche. Per Aristotele, la buona vita o l'eudaimonia consiste nella capacità di sapere quale sia la "cosa giusta" e nella capacità di metterla poi, appunto, in pratica. Nella sua teoria della buona vita, sviluppata nell'Etica Nicomachea, Aristotele parla della buona vita come della vita felice, ma non equipara la buona vita alla mera sensazione di essere contenti o divertiti. Al contrario, la buona vita, per una persona, consiste nella vita attiva del buon funzionamento nei modi essenziali e unici per gli esseri umani. Di conseguenza, permane una "vita attiva dell'elemento che ha un principio razionale" (Aristotele, 1098a). Tale proclamo consta di due parti importanti per la definizione di eudaimonía che dà Aristotele: da un lato, si tratta dell'elemento della "vita attiva", e, dall'altro, dell'elemento del principio razionale (o, in altre parole, del logos) tramite il quale la vita pubblica dovrebbe essere governata.

Aristotele riteneva che la capacità di ragionare fosse una prerogativa degli esseri umani e che, di conseguenza, per gli umani il bene consistesse nella massima realizzazione di tale funzione. La buona vita venne considerata, da parte di Aristotele, come attività dell'anima in relazione alla virtù. Per quanto riguarda lo Stato, Aristotele afferma nella Politica che la società politica esiste non semplicemente per organizzare la vita in comunità, ma "per la ricerca di azioni nobili" (Pol. III. 9.1281a3). Fare il giusto era, per Aristotele, parte dell'essere un cittadino attivo; una persona che partecipava alla vita pubblica della propria comunità e che prestava il proprio aiuto,

1 Il presente saggio ha beneficiato delle ricerche del dott. Ramon Rodriguez-Amat, di Petar Mitric e di Izabella Korbiel. 
modellava il processo decisionale. La democrazia viene quindi ritenuta la massima partecipazione alla vita pubblica. Di conseguenza, lo Stato ha la responsabilità di agire in maniera tale da cercare di garantire una buona vita ai propri cittadini consentendo loro di partecipare appieno alla "cosa" pubblica. La forma diretta di democrazia di cui godevano gli ateniesi non solo permetteva un'amministrazione efficiente dello Stato, ma finanziava la Dracma greca, investiva in grandi opere pubbliche quale la costruzione del Partenone e si rendeva responsabile del benessere dei suoi cittadini.

Di conseguenza, la buona vita dei cittadini richiede uno stato attivo, una cittadinanza attiva, un impegno e un sostegno pubblici. Perseguire la creazione e il sostegno di ambienti che favoriscano il pubblico dibattito costituisce il fulcro dell'azione politica nel mondo, dai movimenti sociali ora conosciuti come la Primavera araba in tempi più recenti ai movimenti sociali per la giustizia globale, da Occupy alle proteste contro l'austerità in tutta Europa. Ciò che unisce tutti questi movimenti è, in ultima analisi, la richiesta di creare le condizioni per esercitare una cittadinanza piena, ovvero partecipare interamente alla vita pubblica e alle politiche dello Stato. La privazione di risorse strutturali e materiali indebolisce la cittadinanza e la democrazia in quanto, senza di esse, qualsiasi legge che preveda i valori di uguaglianza, stato di persona e il diritto alla libertà di associazione rimane priva di fondamenti.

Per garantire una partecipazione significativa dei cittadini alla vita pubblica, devono essere soddisfatte determinate condizioni. Queste condizioni riguardano gli aspetti legali, strutturali, economici, politici, culturali e sociali che circondano la governance della partecipazione politica e la governance della "società politica". Per cominciare, le previsioni giuridiche per la libertà di espressione e i diritti a essa associati devono essere garantite per tutti i cittadini. L'esercizio di tali diritti deve altresì essere facilitato e favorito da parte delle istituzioni sociali nonché da parte dello Stato. Per tale ragione, le limitazioni sproporzionate ed eccezioni eccessive all'esercizio di tali diritti e la riservatezza dello Stato o delle pubbliche amministrazioni non possono essere ritenuti compatibili con lo spirito di libera comunicazione e deliberazione. Inoltre, gli impedimenti strutturali devono essere limitati al minimo possibile affinché si possa ottenere il grado di partecipazione dei cittadini più elevato possibile. Di conseguenza, le questioni educative, la disponibilità e l'accessibilità delle fonti informative, l'equità e l'inclusione rappresentano elementi chiave negli ambienti che favoriscono una tale partecipazione. Inoltre, la responsabilità etica verso la veridicità e la tutela dai sotterfugi e dalla disinformazione rappresentano ulteriori prerequisiti per costruire le condizioni adeguate per le pubbliche deliberazioni. La "situazione discorsiva ideale" di Habermas (1984), per esempio, fa ri- 
ferimento a tali condizioni così come alla libertà dal timore del castigo, affinché tutte le opinioni possano essere espresse. Per Aristotele la creazione della conoscenza, il sentiero verso la conoscenza, sia che si tratti di una ricerca scientifica o che esso risieda nella società politica, passa attraverso la dialettica, ovvero l'esistenza di voci antagoniste e la ricerca del falso in qualsiasi momento. Per tale scopo, Aristotele immagina una libertà di confronto e l'uguaglianza tra le persone, che si distingua o meno dalle loro capacità di applicare il metodo scientifico o meno (Berti 1978). Una dialettica di opinioni è, secondo Aristotele, necessaria per una democrazia e la libertà di espressione risulta, in relazione a tale dialettica, necessaria, non per il diritto di un singolo ma "come contributo del singolo alla realizzazione del bene comune di natura politica o scientifica" (Berti 1978: 369). Di conseguenza, la concezione storica del bene comune che si intenda in relazione al cittadino ci porta ai principi fondamentali che sottendono un sistema di governo. Senza tale partecipazione, la quale, per Aristotele, andrebbe perseguita anche tramite l'abolizione di disuguaglianze economiche estreme, la governance di una società è caratterizzata dalla tirannide.

Questa breve analisi sul legame tra il bene comune, la partecipazione pubblica e la libertà di espressione è utile per porre le basi per un dibattito dettagliato dal punto di vista empirico in merito agli ambienti all'interno dei quali le società europee sono oggi chiamate a difendere le istituzioni democratiche e a rivitalizzare il rapporto tra i cittadini e tali istituzioni. Il pubblico dibattito è la pietra miliare della democrazia, ma soltanto se l'accesso alla sfera pubblica è garantito e consentito a tutti i cittadini e con le diverse voci ed esperienze. In particolare, la richiesta di accesso universale alla sfera pubblica, sia in forma mediatica - tramite i mass media, per esempio - che in forma diretta presuppone varie condizioni, una delle quali è la libertà di espressione. Inoltre, la libertà di espressione dipende da determinanti strutturali e simboliche. Non sono soltanto richiesti spazi per il dibattito accessibili e utilizzabili - per esempio, mezzi di informazione accessibili, tecnologie utilizzabili dagli utenti per la partecipazione, ecc. Essi devono altresì sostenere una cultura di inclusione e rispetto che consenta $\mathrm{e}$ che crei spazi di interazione per gruppi o idee storicamente o altrimenti marginalizzati.

Il presente capitolo analizza i modi in cui le emittenti pubbliche affrontano le loro sfide esistenziali in Europa e quali implicazioni ciò comporta, e comporterà, per la democrazia e la coesione sociale nel continente. Il dibattito colloca le "difficoltà" attuali del servizio pubblico all'interno del contesto della crisi economica nonché il suoi impatto sul giornalismo e sulla libera diffusione delle informazioni e lo sviluppo storico dei controlli di auditing per i media pubblici, i quali sono stati, in generale, sproporzio- 
nati rispetto alle aspettative di responsabilità richieste nei confronti dei media privati. Il pubblico servizio occupa storicamente una posizione unica di organizzazione svolgendo funzioni ininterrotte per quasi un secolo nel continente europeo. Si tratta delle uniche istituzioni incaricate di missioni comprensive di portata universale al fine di fornire informazioni, cultura e momenti di formazione al cittadino. Si tratta altresì degli unici media totalmente in mano pubblica a servizio del pubblico. La questione che verrà dibattuta in seguito porta a chiederci che cosa succede in una società priva del servizio pubblico? Affronteremo il particolare caso della chiusura dell'azienda radiotelevisiva di Stato greca (ERT) da parte del governo greco l'11 giugno 2013. Ci guarderemo poi intorno in Europa tramite un "diffuso" confronto per chiederci se quello di ERT sia stato un caso unico ed estremo, determinato dalle condizioni finanziarie straordinarie dell'economia greca, o se il "caso ERT" potrebbe essere ritenuto il precursore di una tendenza nella governance dei media pubblici, e, in seguito, nella governance del libero dibattito e delle pubbliche organizzazioni nelle società europee.

È necessario analizzare la situazione attuale del servizio pubblico nel contesto di una prolungata e imprevedibilmente persistente crisi finanziaria in Europa. L'ambiente complessivo per un libero dibattito sulle fondamenta democratiche non è causa di gioia, perlomeno se si guarda ai modi in cui i cittadini vedono le istituzioni, e ne misura la fiducia nel ruolo dei media tradizionali nel pubblico dibattito. In verità, un malessere politico ha cominciato a determinare la vita pubblica europea, caratterizzato dalla sfiducia nelle istituzioni, la premeditata costruzione della polarizzazione nelle nazioni europee verso i migranti non-europei, i rifugiati e i richiedenti asilo, insieme ad una debole pluralità di voci che trattano l'argomento. Il malcontento dei cittadini si manifesta negli spazi fisici e digitali tramite proteste, la nascita di una moltitudine di forum mediatici e altri modi di comunicare, spesso bypassando i classici mezzi di comunicazione e ricercando collegamenti diretti tra di loro nel tentativo di reclamare il proprio spazio nelle politiche democratiche, se non per "ricostruire" le politiche democratiche.

\section{LE GRANDI CRISI NEGLI SPAZI COMUNICATIVI IN EUROPA}

La governance degli ambienti comunicativi è strategica in un'epoca in cui vengono attuate politiche pubbliche impopolari e probabilmente incostituzionali per un periodo di tempo prolungato. Ciò avviene chiaramente in Grecia, ma anche in altri Paesi europei. I cambiamenti sul panorama me- 
diatico non sono semplici conseguenze della crisi, bensì piuttosto atti strategici per governare l'opinione pubblica più che per governare la crisi.

Nella periferia europea, dal Regno Unito e l'Irlanda al Portogallo, alla Spagna e alla Grecia, nonostante i diversi contesti economici, il ritiro dello Stato dalla rete di sicurezza sociale e dal welfare e il deterioramento degli standard di vita hanno innalzato onde di protesta e politiche di opposizione. Nonostante i ripetuti "pacchetti", "programmi di bail-out", "misure emergenziali”, ecc., a partire dal 2008 la povertà è aumentata: in media, la popolazione europea a rischio povertà o esclusione sociale è cresciuta dal 23,5\% del 2008 al 24,2\% del 2011. In Grecia l'aumento fu dal 28,1\% al $31 \%$ e in Spagna dal 22,9\% al 27\%. La Spagna conta 12,4 milioni di "poveri” e la Grecia 3,4 milioni (Eurostat, 2012). Il numero dei bambini che vivono in condizioni di povertà è drammaticamente cresciuto nel Regno Unito raggiungendo la soglia più alta, in numeri assoluti, da decenni (DWP 2015). Negli scorsi quattro anni, il tasso di suicidi è aumentato del $45 \%$ in Grecia (Branas et al. 2015). Il suicidio come atto politico e come azione estrema ha raggiunto nuovi livelli. Nel 2012, l'Associazione bancaria spagnola ha annunciato, in risposta all'aumento dei suicidi, che avrebbe congelato gli sfratti in casi di estrema difficoltà (The Guardian 2013b). Secondo la Plataforma de Afectados por la Hipoteca (Piattaforma degli sottoscrittori di un mutuo) vi furono 40.000 sfratti in Spagna a partire dall'inizio della crisi economica nel 2007.

La mobilizzazione sociale attraverso scioperi e proteste è all'ordine del giorno in Europa ed è, come ci si può aspettare, più elevata nei Paesi più duramente colpiti dalla crisi. Si sono moltiplicate le azioni coordinate nate dal basso a livello pan-europeo: gli insegnanti delle scuole e degli asili, i lavoratori che operano nel campo della salute, il personale universitario, i giornalisti e gli addetti alla comunicazione, così come i sindacati generali dei lavoratori si sono mobilizzati. Mostrare solidarietà di fronte a ciò che viene percepito come condizioni di crisi in cui è impossibile lavorare è diventato un elemento permanente nelle città europee, diversamente dai discorsi di segregazione e polarizzazione che si fanno strada tra le élite politiche. $\mathrm{E}$ in questo punto d'incontro cruciale di evidente divario tra i governi e i cittadini d'Europa che è fortemente necessario un solido dibattito pubblico, sostenuto da un forte giornalismo investigativo e da ambienti che consentano la ricerca di strade per uscire da ciò che sembra un circolo vizioso. La mobilizzazione sociale suggerisce che questi spazi per il dibattito siano infatti aperti e vivaci e che, per definizione, i media dovrebbero essere parte integrante di essi.

In ogni caso, le testimonianze sono motivo di preoccupazione: alcune organizzazioni internazionali hanno lanciato l'allarme in merito agli aspet- 
ti più importanti della libertà di parola. Dall'inizio della crisi nel 2009, Reporter senza frontiere ha messo in guardia dal declino della stampa europea nella classifica della libertà di espressione. Nel 2013 ha registrato una polarizzazione nelle direzioni intraprese da vari Paesi secondo il livello di libertà di stampa di cui godono; alcuni hanno mantenuto la loro posizione, quali la Finlandia, la Germania e l'Austria, mentre altri hanno rapidamente perso terreno, quali l'Ungheria, la Grecia, l'Italia e persino la Francia. I fattori che hanno contribuito a queste cadute sono una combinazione tra un susseguirsi di riforme giuridiche e mutamenti drastici, la posizione politico-economica dei media e l'aumento di forme informali di governance tramite reti di interesse. Le valutazioni globali annuali a cura di "Freedom House" hanno evidenziato un punto mai così basso nella storia della libertà del mondo e standard decrescenti di libertà nelle democrazie mature. L'indice sulla censura ha mostrato nel dettaglio gli innumerevoli casi di censura, violenza e intimidazione contro gli operatori mediatici e i giornalisti che tentano di far emergere punti di vista critici.

L'OSCE, nel 2007, ha affermato che quasi la metà dei suoi 56 Paesi membri ha imposto sanzioni giuridiche contro i giornalisti che ottengono e pubblicano informazioni confidenziali. Il fenomeno si è manifestato maggiormente nei Paesi dell'est e del centro Europa, laddove molti Sati hanno introdotto nuove leggi sui segreti di Stato, quali la Repubblica Ceca, la Moldavia, la Bulgaria, l'Albania e la Croazia. In questo contesto, Banisar rivela "una tendenza importante nell'uso di leggi sui segreti di Stato per penalizzare i whistleblower e i giornalisti che pubblicano informazioni di pubblico interesse" (Banisar 2008:15). Tali casi sono stati rilevati in Danimarca, Croazia, Bulgaria, Romania, nel Regno Unito, in Germania e in Svizzera.

Nel frattempo, in termini molto concreti, l'austerità e la crisi hanno duramente colpito la già precaria professione del giornalismo. Strettamente collegata al mutamento dei panorami comunicativi è la perdita di lavoro e la crescente precarietà per l'ampia maggioranza di giornalisti attivi. Le condizioni di lavoro per i giornalisti si sono drammaticamente trasformate nell'ultimo decennio e la crisi ha esacerbato tale sviluppo. In Spagna, per esempio, El País ha riportato nel 2012 che più di 70.000 giornalisti erano senza lavoro - il triplo dell'anno precedente (El País 2012b). Soltanto il Gruppo Prisa ha licenziato 1.281 impiegati nel 2012 (20 Minutos 2012). In Grecia la disoccupazione a lungo termine è ormai diventata la norma e, inoltre, la maggior parte dei posti di lavoro disponibili sono precari, con un salario mensile medio di 400 euro, spesso richiedendo che gli articoli vengano scritti in forma anonima. Vi sono almeno 2.500 giornalisti senza lavoro in Grecia, secondo POESY, l'associazione che raccorda tutti i sinda- 
cati del Paese. Questa rappresentazione è imprecisa dal momento che include soltanto i giornalisti registrati in un sindacato. La registrazione non è automatica se non è stato svolto un determinato numero di ore nei media. Dato che la maggior parte delle industrie dei media assume soltanto giornalisti freelance, ciò significa che all'ampia maggioranza di giornalisti, in particolare i giovani, è automaticamente preclusa l'iscrizione a un sindacato. Esiste quindi una situazione paragonabile alla trama del romanzo "Comma 22": è praticamente impossibile, per un giornalista freelance, registrarsi e accreditarsi come giornalista, ma senza l'accreditamento pieno i giornalisti e i fotoreporter sono obbligati a lavorare in un regime di incertezza e rischiano la vita in situazioni pericolose, per esempio quando riportano le proteste in Grecia. Il risultato di ciò è, come afferma Reporter senza frontiere, che i giornalisti sono sempre più indifesi in situazioni di malcontento sociale e negli scontri con la polizia. Gli indici sulla censura e Reporter senza frontiere hanno definito la situazione in Grecia esplosiva: hanno paragonato il report delle proteste nel Paese ai report di guerra a causa della violenza e dell'abuso di potere da parte della polizia, la quale prende di mira i giornalisti e li attacca non soltanto in modo indiscriminato, ma anche strategico. Amnesty International ha pubblicato un rapporto sulla polizia greca, affermando che essa opera in una cultura dell'impunità e della violenza (Amnesty International 2012).

Sulla problematica dell'impunità della polizia nei casi di attacchi contro i giornalisti, il Report sull'impunità del 2014 contiene riferimenti ai giornalisti le cui vite sono a rischio. Una combinazione di fattori contribuisce al precario stato di salute e di sicurezza dei giornalisti professionisti, inclusa la mancanza di investigazione nel caso di attacchi e intimidazioni poliziesche, la criminalizzazione dei giornalisti e le politiche di Stato sulla sicurezza, la mancanza di trasparenza e la manipolazione Secondo Freedom House, la Grecia ha vissuto il peggiore e più ampio divario di punti tra il 2009 e il 2013, posizionandosi dietro il Bahrain, l'Ucraina, l'Egitto, il Kazakhistan e l'Azerbaigian nella categoria dei Paesi "in parte liberi". La chiusura di ERT ha contribuito a questo effetto dannoso complessivo.

Nonostante la condizione critica del mercato del lavoro per gli operatori dei media non costituisca il punto centrale della presente trattazione, essa ci serve a comprendere il ruolo cruciale dei media pubblici in un ambiente segnato da instabilità e precarietà. I media pubblici hanno storicamente funto da punti di riferimento stabili in questo mercato, soprattutto quando il mercato impone salari da 200 euro al mese per scrivere 28 articoli al giorno (Milanuncios 2013). Nel caso della Grecia, dove l'interesse di implementare le misure di austerità - in opposizione alle varie misure che puntano meno sulla riduzione dei salari e sulle privatizzazioni - sono molto alte, 
il controllo governativo del settore pubblico viene effettivamente applicato tramite intimidazioni, al di sopra del contesto esistenziale all'interno del quale la maggior parte dei giornalisti si ritrova. Le opportunità di lavoro per i giornalisti giovani vengono create tramite il licenziamento sistematico dei giornalisti più anziani dissenzienti come nei casi precedentemente citati dei media pubblici in Grecia e in Spagna. Altri esempi sono il giornalista greco Kostas Vaxevanis, premiato a livello internazionale, della rivista investigativa Hot Doc, arrestato per aver pubblicato ciò che è divenuto noto col nome di "Lagarde List" dei greci benestanti contro i quali era stato suggerito al governo greco di indagare per evasione fiscale (Smith 2012); o Lefteris Charalampopoulos, reporter dell'Unfollow Magazine greco, minacciato dopo aver pubblicato i sospetti che Aegean Oil fosse coinvolta in uno scandalo di contrabbando (Zenakos 2013). Gli attacchi fisici che contengono un grado straordinario di violenza da parte della polizia greca sono altresì stati ben documentati, specialmente nel caso di Mario Lolos, il capo dell'Unione dei fotoreporter greci, rimasto vittima di disabilità permanente (Indice sulla censura). Altri giornalisti che si erano chiaramente identificati nei confronti della polizia vennero altresì aggrediti e necessitarono di trattamenti ospedalieri. Attacchi del genere sono accaduti anche altrove. In Spagna, per esempio, alcuni quotidiani internazionali hanno riferito di un'epurazione di giornalisti che avevano posto domande scomode (Murado 2012 e Baboulias 2012).

\section{IL QUARTO MODELLO DI GOVERNANCE DEL SERVIZI PUBBLICO? DALLA CHIUSURA DI ERT A ERTOPEN}

In questo clima venne annunciata, l'11 giugno 2013, la decisione del governo greco di chiudere ERT in modo arbitrario e senza approvazione da parte del Parlamento. La chiusura venne ordinata tramite un Decreto speciale il quale avrebbe richiesto, in teoria, la convalida da parte del Parlamento greco, ma al Parlamento non fu mai data la possibilità di votarlo. Oltre 2.500 professionisti altamente qualificati, tra cui molti giornalisti, si aggiunsero, con effetto immediato, all'alto numero di disoccupati. Gli schermi della televisione greca si oscurarono in quello che in Grecia ed a livello internazionale divenne noto come "L'oscurità". Gli ex dipendenti di ERT occuparono le sedi di ERT ad Atene e Salonicco (il quartier generale di ERT3, la più grande emittente radiotelevisiva la cui sede non si trova ad Atene e che trasmette in tutta la Grecia). Per vari mesi dopo l'11 giugno 2013 i cittadini trasformarono le sedi ERT di Atene e Salonicco in luoghi di pubblico dibattito che vennero trasmessi da parte degli ex dipendenti di ERT. 
La decisione spontanea di proseguire a trasmettere si trasformò in un'azione politica a lungo termine che avrebbe poi riportato ERT sugli schermi televisivi greci: l'11 giugno 2013 il neoeletto governo di Syriza ottenne l'approvazione del Parlamento per una nuova cornice giuridica che permise a ERT di riaprire. Nel momento in cui questo capitolo è stato scritto, ERT stava trasmettendo un palinsesto completo, anche se temporaneamente, senza essere ritornato ai precedenti livelli di trasmissione.

La decisione di chiudere ERT venne annunciata dal portavoce del Governo Simos Kedikoglou, egli stesso un ex dipendente di ERT, citando "la corruzione cronica e la mala gestione" dell'azienda (Kedikoglou 2013 e Euronews 2013). Ciò avvenne nonostante le categoriche smentite da parte di Kedigoglou sul proprio sito web del 15 e del 19 maggio che ERT stesse per essere chiusa. Apparentemente, il sito web è stato oggetto di un attacco informatico e non è più raggiungibile. "ERT rappresenta un caso di eccezionale mancanza di trasparenza e stravaganza incredibile. Tutto ciò ora finisce", disse il portavoce del governo Simos Kedikoglou durante una conferenza stampa (BBC News 2013). Circa un mese prima, Kedikoglou aveva smentito in varie interviste le voci circa un'imminente chiusura di ERT:

- In particolare fu Kedikoglou a smentire, in svariate circostanze, categoricamente che ERT fosse prossima alla chiusura, anche nelle interviste del 15 e del 19 maggio, così come sul proprio sito web (che è stato apparentemente oggetto di attacco informatico e non è ora più raggiungibile).

- La chiusura di ERT ha immediatamente scatenato dei dubbi su vari articoli della Costituzione greca, così come del Trattato di Amsterdam, il quale supervisiona le trasmissioni pubbliche in Europa (Nevradakis 2013).

Queste dichiarazioni contraddittorie ricordano le affermazioni di Psychogiopoulou et al. (2011:4) secondo cui "la politica mediatica greca è stata caratterizzata per anni da un modello decisionale essenzialmente non trasparente e governo-centrico". La chiusura ha provocato una diffusa condanna internazionale da parte delle associazioni di categoria, l'EBU, il Parlamento Europeo e varie ONG, per esempio Reporter senza frontiere. TEXTE, la rivista pubblicata dalle aziende del servizio pubblico di Austria, Germania e Svizzera ha dedicato agli eventi in Grecia un proprio numero (Mischka \& Unterberger 2013). Otto mesi dopo la chiusura, digitando "ERT" su un motore di ricerca internet si trovavano oltre un milione di menzioni nei social network.

La Commissione europea affermò che intervenire in questioni nazionali non rientri all'interno del proprio ambito di competenze e richiese la ria- 
pertura di ERT (Commissione europea 2013). In Grecia, il Consiglio di Stato, il massimo organo di giustizia amministrativa, ordinò immediatamente al governo di ripristinare ERT, ma tale ordine venne ignorato. I sindacati che rappresentavano il personale di ERT avviarono immediatamente delle cause, separatamente per ogni area geografica, contro il governo a causa dell'illegittima e incostituzionale chiusura. La prima sentenza, pronunciata a Candia, Creta, dichiarò il licenziamento dei dipendenti di ERT incostituzionale e ordinò al governo di riassumerli e di ricompensarli con effetto immediato. Il governo non fece nulla. Sentenze simili vennero emesse anche in altri casi in Grecia. Nel frattempo, il governo venne costretto, sotto pressioni internazionali, a costituire un servizio pubblico di transizione denominato Dimosia Tileorasi (DT), al costo di oltre un milione di euro al mese. DT diede adito a un nuovo servizio pubblico, chiamato NERIT, anch'esso, come DT, sotto la diretta supervisione del Ministero delle finanze. NERIT proseguì le proprie trasmissioni fino al 10 giugno 2015, il giorno in cui ERT venne ristabilito. Il processo di reclutamento di personale per NERIT si rivelò problematico, a causa della mancanza di trasparenza. La dirigenza si dimise e vari scandali coinvolsero NERIT, inclusa l'infame "intervista" televisiva dell'allora Primo Ministro, Antonis Samaras, da parte di due giornalisti di NERIT, nella quale Samaras lesse le proprie risposte alle domande di un suggeritore elettronico. Per molti osservatori questo incidente evidenziò la differenza tra NERIT ed ERT.

Non soltanto ERT si auto-finanziava chiedendo agli spettatori un canone mensile di circa 4 Euro a famiglia, ma nel 2010 era riuscito a saldare i propri debiti ed a generare un avanzo di circa 100 milioni di Euro. Nel momento della sua chiusura, di conseguenza, non solo non causava oneri al governo greco, ma aveva già proposto di utilizzare i propri fondi per finanziare programmi di assistenza sociale a livello nazionale, quale la riabilitazione di tossicodipendenti, ma il governo rifiutò tale proposta. ERT ha subito recentemente vari tentativi di ristrutturazione. Pur con le peculiarità in cui si differenziano ERT e RTVE (il servizio pubblico spagnolo) i governi hanno, indipendentemente dalle loro ideologie, considerato le aziende di pubblico servizio strumenti a loro disposizione. Nei due anni che precedettero la chiusura di ERT, il suo Direttore Generale, Emilios Liatsis, che era stato nominato dal governo greco, esercitò una censura estensiva sui programmi e finì nel mirino delle critiche per essersi circondato da uno staff di segretarie superpagate (Reporter senza frontiere 2011). Nello stesso periodo il governo intraprese alcuni tentativi per evitare che i giornalisti dello show televisivo mattutino trattassero questioni importanti quali l'uso della violenza da parte della polizia e l'impatto delle politiche di austerità. Il livello di interventismo politico in ERT portò a manifestazioni di preoc- 
cupazione in relazione all'articolo 19 (2012), specialmente nel caso dell'allontanamento di Marilena Katsimi e Kostas Arvanitis dal programma televisivo mattutino per aver espresso la loro contrarietà alle dichiarazioni del Ministro dell'Ordine pubblico in relazione ad una manifestazione antifascista.

Durante i due anni trascorsi tra la chiusura e la riapertura di ERT, il pubblico servizio continuò a trasmettere basandosi su un modello di autogoverno, inizialmente dai propri studi di Atene e poi dagli studi di ERT3 a Salonicco. Queste trasmissioni prevedevano programmi d'informazione radiofonica orari in 16 delle 19 emittenti radio regionali. Le trasmissioni televisive vennero spostate da Atene a Salonicco nel novembre 2013 in seguito ad un'agitazione causata dalla squadra antisommossa della polizia negli studi di Atene. Nonostante la disattivazione del servizio digitale, ERT utilizzò "tattiche da guerriglia" per mantenere in funzione i propri trasmettitori analogici. Tra il 50\% e il 70\% delle abitazioni ubicate al di fuori della città di Atene furono in grado di ricevere le trasmissioni radiotelevisive durante i due anni in questione. Inoltre, ERT continuò a trasmettere su internet tramite ERTOpen (ancora adesso, nel momento in cui si scrive, in diretta su www.ERTopen.com, mentre il servizio online completo è stato riattivato il 29 giugno 2015 su www.ert.gr).

Nonostante l'obiettivo del presente capitolo non consista nel descrivere nel dettaglio il modo in cui ERT ha sviluppato un sistema di autogoverno, è importante fare riferimento ad alcuni elementi cardine di questa esperienza. Nelle prime settimane dopo la chiusura, ex dipendenti, ora stranamente simili a trasmettitori "pirata", seguirono un modello di autodisciplina continuando a rimanere operativi nelle loro precedenti posizioni (tecnici, giornalisti, ecc.), nonostante ci fosse la consapevolezza che le vecchie gerarchie non valevano più. Nei 24 mesi a seguire, molti lasciarono ERT per varie ragioni, inclusa una ragguardevole minoranza che si candidò per ottenere un impiego in DT e poi NERIT. Coloro che decisero di rimanere, scelsero di riorganizzare il modello di governance di ERT aggiungendo un consiglio dei dipendenti, dei sindacati ed assemblee a livello generale e regionale, le quali presero le decisioni sui programmi da trasmettere ed il finanziamento di coloro che continuarono a produrre un determinato programma, dall'acquisto di beni consumabili (nastri, hardware, ecc.) al pagamento delle spese per consentire alle squadre di viaggiare da Atene a Salonicco per fornire il supporto ai telegiornali quotidiani. I fondi arrivarono dal personale stesso, tramite donazioni di ex colleghi e, più avanti, da contributi provenienti dal tutto il mondo, inclusi gruppi di solidarietà di cittadini ed eventi di raccolta fondi. 
Le decisioni sui programmi radiotelevisivi, incentrati su un notiziario quotidiano, vennero prese su base settimanale. Il personale, inclusi i caporedattori, assunse i vari incarichi a rotazioni. Tutti coloro che furono coinvolti nel mantenere la trasmissione di ERT dovettero acquisire, e impartire, nuove competenze per sostenere la produzione del telegiornale. Quando vennero aggiunti nuovi programmi, questa attività venne estesa a gruppi sociali e membri della società i quali, con il supporto di professionisti, crearono nuovi programmi gestiti quasi interamente da non-professionisti. Quando i mesi diventarono anni, queste conoscenze vennero sviluppate tra i più giovani, quali neodiplomati o giornalisti volontari per i media della comunità, così come altri soggetti interessati e capaci a svolgere un progetto, quale la produzione di un programma a cadenza regolare. Immediatamente, l'apertura di ERTOpen e, in particolare, di ERT3 attirò movimenti sociali e gruppi della società civile, i quali fornirono informazioni e risorse. Non è un esagerazione affermare che ERT3 divenne un punto di riferimento cardine per una moltitudine di attori e gruppi sociali, specialmente per coloro che erano coinvolti nel fornire una specie di servizio pubblico, quali gli insegnanti, gli operatori sociali, gli addetti alle pulizie del settore pubblico e addirittura attivisti dell'ambiente, per esempio il movimento "AntiGold" e l'Iniziativa dei cittadini europei "Save the Water".

Il risultato di questi nuovi legami con la società in senso più ampio consistette in un canale di comunicazione diretto che assicurò l'input di risorse per il sostegno dell'organizzazione. Il modello di autogoverno sviluppato in questi due anni è forse unico nella storia dei media europei, ma certamente non sconosciuto, se pensiamo alle iniziative dei media di comunità sudamericane o altre imprese autogestite e dirette dai lavoratori nel mondo. Infatti, esempi del genere mostrano che la garanzia di professionalità e di qualità, da un lato, e l'adempimento delle competenze del pubblico servizio dall'altro, sono funzioni che possono essere svolte appieno tramite modelli di governo democratici che non dipendono da strutture permanenti e gerarchiche. L'"esperimento vivente" di ERT dimostra in modo pratico che un approccio pragmatico per assicurare l'accettanza piena del pubblico servizio da parte della società e la legittimazione dello stesso all'interno della medesima avviene tramite il nucleo dell'organizzazione del lavoro quotidiano. Liquidare tali modelli come utopici o irrealistici, specialmente quando si ha a che fare con un'organizzazione competente con migliaia di dipendenti, è segno di incomprensione. Non soltanto un modello di governace del genere è in grado di fornire un contributo significativo in termini di approccio filosofico al presente ed al futuro del pubblico servizio, può altresì offrire soluzioni ai problemi che le grandi organizzazioni si trovano ad affrontare. Rimane da vedere se, e in quale misura, la 
recente ricostituzione di ERT potrà resistere alle pressioni di ritornare alla percepita normalità della sua precedente incarnazione.

La riapertura di ERT ha attirato il medesimo interesse, sia in Grecia che a livello internazionale, della sua chiusura. Guardando indietro, quali furono le ragioni e l'impatto della sua chiusura? Il ragionamento del governo prevedeva di ripulire l'organizzazione e di creare una "BBC greca". Le accuse di mala gestione e di erogare stipendi troppo elevati per i dipendenti si rivelarono fuorvianti. I notiziari di ERT erano stati in grado di mantenere il loro carattere investigativo e di porsi degli interrogativi sugli sviluppi nel Paese, in particolare sull'impatto della crisi sui gruppi sociali, nonostante i tentativi del governo di esercitare maggiore controllo su di essi. Durante l'assemblea del suo partito, Nuova Democrazia, nel 2013, l'allora Primo Ministro Antonis Samaras affermò, nel corso del proprio intervento, che ogni governo aveva approntato le proprie modifiche a ERT, ma che tutti gli impiegati assunti autonomamente da ERT erano dei "comunisti". Molti osservatori compresero che alla chiusura di ERT da parte del governo Samaras sottostavano ragioni politiche. In ogni caso, è probabile che un altro motivo fosse dato dalla vendita dei diritti di trasmissione digitale in Grecia. Il bando per la ricerca di partecipanti al progetto venne pubblicato dal governo solo pochi giorni dopo la chiusura di ERT e, senza che ERT potesse partecipare alla gara, l'appalto fu facilmente aggiudicato da DIGEA, un'iniziativa imprenditoriale congiunta delle principali cinque imprese del settore mediatico della Grecia.

\section{LA CONDIZIONE DEI MEDIA DEL SERVIZIO PUBBLICO IN EUROPA: UN MODELLO DI SMANTELLAMENTO?}

La chiusura forzata del servizio pubblico greco potrebbe sembrare, in un primo momento, un caso estremo o semplicemente una "tempesta in un bicchier d'acqua". Tuttavia, uno sguardo più attento verso altri Paesi europei mostra che le chiusure e l'imposizione di restrizioni ai servizi pubblici europei stanno rapidamente diventando la norma. Poco dopo la chiusura forzata di ERT in Grecia, il governo spagnolo ha deciso di chiudere il servizio pubblico valenciano, ed il governo israeliano ha proseguito chiudendo il proprio servizio pubblico. Nonostante anche queste chiusure potrebbero essere ritenute casi estremi, è importante analizzare gli sviluppi avvenuti all'interno delle risorse strutturali del servizio pubblico in Europa.

La Grecia e la Spagna non sono i soli due Paesi alla periferia dell'Europa che hanno dovuto fronteggiare difficoltà economiche. Entrambi i Paesi vennero governati da dittature nella loro storia recente e la libertà di 
espressione occupa uno spazio particolare nella memoria popolare e nella cultura. Storicamente ERT in Grecia e RTVE in Spagna furono aziende di Stato. Durante la dittatura entrambi i servizi pubblici nazionali vennero impiegati come strumenti di propaganda: per esempio, il canale greco ERT2 nacque negli anni 60 come YENED e venne gestito e controllato dall'esercito greco. La TV pubblica spagnola sorse durante la dittatura franchista su raccomandazione dei ministri liberali interessati allo sviluppo economico del Paese. Solo negli anni 90 il servizio pubblico spagnolo si evolse dal punto di vista tecnologico e cominciò a rivolgersi ad un pubblico internazionale. Nel decennio scorso, il deficit del servizio pubblico spagnolo ha portato a vari mutamenti nella propria organizzazione: nel giugno del 2006, l'ente pubblico RTVE e le proprie aziende TVE S.A. (TV di Stato) e RNE S.A. (radio di Stato) si fusero creando RTVE. La nuova azienda ridusse poi il numero dei propri dipendenti di 4.855 unità diventando il servizio pubblico più piccolo d'Europa. Le misure di austerità adottate in Spagna in seguito alla crisi economica fornirono inoltre un espediente al governo conservatore per esercitare pressioni su RTVE affinché l'azienda si liberasse di giornalisti ed intervistatori esperti e critici in grado di porre ai politici domande "vere" (ovvero imbarazzanti) (Murado 2012). La dirigenza di RTVE ha sostituito questi giornalisti ed intervistatori con membri del Partito Popolare, che allora si trovava al governo (Burgen 2012).

La chiusura di parti e servizi di RTVE fu attuata tramite privatizzazioni, meno produzioni in-house e il licenziamento di una parte del personale. Il governo introdusse nuove argomentazioni per legittimare i propri cambiamenti nella dirigenza di RTVE, affermando che ciò avrebbe consentito all'azienda di prendere decisioni "in modo più rapido ed efficiente" e di ridurre ulteriormente la spesa (BOE 2012:30986). I cambiamenti, comunque non ai livelli della severità applicata in Grecia, comportarono l'inserimento della Spagna nella lista dei Paesi europei che hanno ridotto la portata e la funzione del loro servizio pubblico su vari piani. Il principale cambiamento apportato dal Partito Popolare nella governance di RTVE consistette nel conferire al governo del diritto di nominare il Direttore generale di RTVE senza l'approvazione del Parlamento. Il Regio Decreto 15/2012 (BOE 2012:30985) che rese esecutiva questo cambiamento venne approvato dal Parlamento, nel quale il Partito Popolare disponeva di un'ampia maggioranza. Il Partito Popolare giustificò la scelta affermando che il procedimento precedente era "visibilmente inefficace in quanto non consentiva il rinnovo della dirigenza con la necessaria agilità per evitare la paralisi del normale funzionamento dell'azienda" (BOE 2012:30986). Il cambiamento venne accompagnato dalla seguente affermazione del governo: "In relazione al settore pubblico, del quale l'azienda RTVE fa parte, il Governo ha as- 
sunto un impegno per ottenere la massima austerità ed efficienza e sta attualmente attuando un processo di razionalizzazione" (BOE 2012:30985). Allo stesso modo, il preambolo del Regio Decreto afferma chiaramente che gli obiettivi del cambio di governance consistono nell'applicare tagli al bilancio e nel fornire a RTVE una dirigenza che attuerà tali cambiamenti.

Il medesimo Regio Decreto consentiva al governo di chiudere le reti di servizio pubblico nelle regioni autonome privatizzando i loro servizi (Congreso Diputados 2012). La stampa ha riportato questa nuova politica come una misura di austerità volta a "flessibilizzare" il modello del servizio pubblico regionale. Se le Comunità autonome scelgono di mantenere i loro servizi pubblici, possono gestirli direttamente o indirettamente, a condizione che i gestori del servizio pubblico attuino i tagli alla spesa (ABC 2012). Ciò significa, in realtà, che i servizi pubblici regionali sono aperti alla privatizzazione (El País 2012a). L'impatto di un'operazione del genere significherebbe probabilmente anche la distribuzione di contenuti centrali e uniformi nelle regioni. Nell'agosto del 2012, alcuni servizi regionali iniziarono a cessare la propria attività: il primo fu "7 Region de Murcia" dopo sei anni di pubblico servizio, per ridurre i costi. In Catalogna i dipendenti dell'azienda di servizio pubblico catalana si opposero alla privatizzazione ed ai tagli. Il Parlamento regionale catalano venne obbligato a instaurare una commissione per gestire i cambiamenti (Parlament de Catalunya 2013:324). I rappresentanti dei lavoratori evidenziarono che i dipendenti di rango inferiore del pubblico servizio, i cui stipendi vengono pubblicati, stavano affrontando una riduzione del $35 \%$ del loro salario, mentre i loro colleghi più pagati, i cui stipendi sono coperti da segreto, non dovevano affrontare una riduzione del salario (Parlament de Catalunya 2013:324). I sindacati denunciarono i tagli al bilancio del governo come parte di una strategia complessiva per smantellare il servizio pubblico catalano. Da parte sua, il Governo regionale catalano affermò che i cambiamenti al servizio pubblico catalano erano semplicemente volti a semplificarne la struttura ed esternalizzare una parte più ampia del lavoro (Parlament de Catalunya 2013:5).

La crisi economica offrì un quadro dialettico e normativo all'interno del quale i governi presentarono misure per tagliare i beni e i servizi pubblici, e le aziende radiotelevisive furono le prime a subirne i tagli. Il governo olandese, formato da una coalizione tra il partito liberal-conservatore VDD ed il partito socialdemocratico PvdA, provocò ampie reazioni quando annunciò la propria decisione di tagliare i fondi al servizio pubblico olandese (NPO) di 100 milioni di euro nel 2016, in seguito ad un taglio di $200 \mathrm{mi}$ lioni nel 2011 ed una riduzione degli introiti complessivi di NPO di un terzo. La conseguenza di questi tagli al bilancio fu una riduzione del nu- 
mero dei canali da 22 a 8 (European Broadcasting Union EBU 2013a). Il governo olandese affermò che queste misure avrebbero reso il servizio pubblico "più semplice e più efficiente" (Governo olandese 2013). Il NPO ha affermato di non poter ovviamente lavorare in queste condizione ed ha esortato, in una mossa altamente inusuale, l'opinione pubblica a scendere in piazza ed a protestare contro questi tagli (RNW 2010).

I servizi pubblici non sono mere unità di trasmissione o canali televisivi. Anche i bilanci dei loro cori pubblici e delle loro orchestre vennero dimezzati. Inoltre, il "Servizio mondiale" dell'NPO (Public Radio Netherlands Worldwide) divenne parte del Ministero degli Affari esteri, a fronte di una riduzione di bilancio del 70\%. L'EBU ha stimato che questi tagli indeboliranno seriamente il NPO, il quale è attualmente "l'emittente più fidata" $\mathrm{e}$ detiene lo share più elevato nel Paese. Si dovrebbe aggiungere che i piani per ridurre la portata e l'ampiezza del lavoro prodotto da NPO facevano già parte di un piano strategico del 2013 che era poi stato abbandonato in seguito al cambio di governo.

Nel frattempo, vi sono stati due tentativi di chiusura di Radio e Televisao de Portugal (RTP) nel decennio scorso. Il dibattito è sorto quando il Ministro degli Affari parlamentari ha annunciato la possibile privatizzazione di RTP (Pfanner 2012), e si è concluso quando RTP ed il Governo portoghese hanno siglato un nuovo contratto all'inizio del 2014. RTP rimane uno dei maggiori servizi pubblici sottofinanziati in Europa. Nel 2013 il suo bilancio annuale venne tagliato di 30 milioni di euro (EBU 2013c). Durante le trattative per il nuovo contratto, l'intera dirigenza di RTP si è dimessa per protesta e l'EBU ha scritto una lettera al Primo ministro Passos Coelho affermando che:

Affidare la gestione di beni preziosi dello Stato a interessi commerciali - un passo senza precedenti nel mondo - metterebbe a rischio la reputazione guadagnata da RTP a partire dal 1974 ... Gli interessi economici e pubblici si mischierebbero ed il pluralismo sarebbe in pericolo. I cittadini potrebbero perdere per sempre un punto di riferimento fidato (Pfanner, 2012).

Il dibattito su come raggiungere gli obiettivi di bilancio includeva la privatizzazione o la chiusura di RTP2, "ristrutturare il ventaglio di attività e servizi offerti dall'azienda e, in aggiunta, ridurre il numero di dipendenti”, oppure vendere una concessione di gestione di RTP1 ad un'azienda privata, finanziata con l'attuale canone televisivo. Alcuni degli oppositori politici di Coelho hanno ritenuto la tentata privatizzazione di RTP un mezzo per limitare le critiche mediatiche al governo di Passos Coelho (Pfanner, 2012). 
Nel Nord Europa venne ordinato al Primo Canale Baltico (PBK) di sospendere la trasmissione dei propri programmi in lingua russa (circa il 70\% del totale) per un periodo di tre mesi. Il PBK è uno dei canali televisivi più sanzionati in Europa e motivo di preoccupazioni internazionali. Il Segretariato dell'OSCE per la libertà di stampa ha affermato che "una misura del genere mette a repentaglio il pluralismo mediatico e deve essere limitata ai casi di pericoloso e intenzionale incitamento alla violenza" (OSCE 2013).

Nel 2013 il Direttore Generale dell'EBU Ingrid Deltenre ha criticato il governo rumeno per le eccessive interferenze politiche nel pubblico servizio del Paese: "Mentre la radio rumena è un forte membro dell'EBU, si potrebbe dire lo stesso della TV rumena se solo il suo amministratore delegato avesse il tempo necessario per dare efficacia alle riforme". Facendo presente che solo uno dei dirigenti della TV rumena è potuto rimanere in carica per l'intero suo mandato, Deltenre ha proseguito affermando che "il Parlamento ha frequentemente utilizzato i due report annuali sull'emittente come espediente per liberarsi dei dirigenti dell'azienda - spesso per evidenti ragioni politiche" (EBU 2013b).

Uno dei casi più problematici in Europa è rappresentato dall'Ungheria, la quale è stata definita un "sistema mediatico non libero" nel 2011 a causa delle sue nuove leggi sui media (Brouillette 2012). Allora l'EBU fece appello al Primo Ministro Viktor Orbán affinche assicurasse il pluralismo mediatico (EBU 2011). L'EBU gli chiese di “dare risposta alle preoccupazioni (dell'EBU) in merito alle minacce alla libertà, all'indipendenza ed al pluralismo dei media in Ungheria scaturite dalla nuova legge". La legge sulla stampa e sui media (legge 104/2010 sulla libertà di stampa e le norme fondamentali sui contenuti mediatici) e la legge sui media (legge 185/2010 sui servizi mediatici e sui mass media) venero adottate sei mesi dopo l'elezione di Orbán. Il comitato Articolo 19 ha emesso un report in cui si esprimono preoccupazioni sulla perdita di indipendenza del servizio pubblico ungherese ed il suo contributo al deterioramento della situazione dei media in Ungheria (Articolo 19 2011a, 2011b).

Nel frattempo, la Telewizja Polska (TVP), il servizio pubblico polacco, ha trasferito, nel 2014, 411 dei propri impiegati a Leasing Team, un appaltatore privato. Tra i 411 dipendenti trasferiti vi furono 116 caporedattori e circa 270 giornalisti. Questa riorganizzazione venne decisa dal direttivo del TVP il 15 aprile 2014 per migliorare la situazione finanziaria precaria dell'azienda (solo il 15\% degli introiti di TVP proviene dai contributi pubblici). Il Presidente del C.d.A. di TVP, Juliusz Braun, aveva riferito di un disavanzo di oltre 220 milioni di PLN (53 milioni di euro) nel 2012, che si ridusse poi a 20 milioni di PLN (4,8 milioni di euro) nel 2013. Nel 2014, per la prima volta dopo tanti anni, TVP registrò un avanzo di 6 milioni di 
PLN (1,45 milioni di euro). Secondo Braun, TVP aveva risparmiato in totale 300 milioni di PLN (72 milioni di euro). Nel 2011, il numero di dipendenti di TVP era stato ridotto da oltre 4.000 a 2.838 , anche allora tramite una "misura di prestito". Come nella maggior parte dei casi, le ragioni che sottendono a tali decisioni non furono ben chiare e basate su procedure di valutazione discutibili. Il personale rimasto di TVP vide cambiare le proprie posizioni da direttori specializzati, reporter e responsabili di sezione a "coordinatori" generali. Le descrizioni delle loro mansioni vennero altresì cambiate in una vaga competenza di "produzione e trasmissione di notizie", senza ulteriori specificazioni. Molti sindacati di TVP e molti ex impiegati hanno fatto causa a TVP ed all'azienda privata, asserendo che il trasferimento di dipendenti all'appaltatore fosse illegittimo, in quanto i diretti interessati non vi avevano acconsentito. Quando TVP offrì ai propri giornalisti la possibilità di ottenere contratti freelance, solo alcuni accettarono: la maggioranza rifiutò (Pytlakowski 2015).

Più recentemente, l'EBU ha espresso un monito sulla situazione critica, "vicina al collasso", di HRT, il servizio pubblico radiotelevisivo della Bosnia ed Erzegovina. HRT è formata da Radio-Televizija Federacije BiH (RTFBiH), che trasmette nella Federazione di Bosnia ed Erzegovina, e da Radio-Televizija Republike Srpske (RTRS), che trasmette nella Repubblica Serba di Bosnia ed Erzegovina. Le ragioni indicate consistono in un cronico e acuto sottofinanziamento e nelle interferenze politiche esercitate dal governo del Paese. Anche l'OSCE ed i rappresentanti dell'UE in Bosnia ed Erzegovina avevano espresso simili preoccupazioni. Da una valutazione più attenta emerge chiaramente che HRT non è stato adeguatamente finanziato per anni, non ricevendo contributi adeguati né beneficiando di un nuovo piano finanziario, che era stato proposto, ma mai adottato. Ciò rappresenta un disastro, tenendo conto del significato di un servizio pubblico funzionante in maniera adeguata per collegare e facilitare il dialogo tra i vari gruppi etnici che convivono in Bosnia ed Erzegovina ed in prospettiva dell'ampio sostegno pubblico delle riforme del servizio pubblico del Paese (UNDP 2014).

Questa rapida analisi del servizio pubblico e dei media pubblici in Europa ha messo in luce uno schema di cambiamento e di sfide per le istituzioni, esacerbate dalle pressioni politiche nel tentativo di affrontare le sfide della crisi economica. I media pubblici non solo hanno subito, essendo spazi di comunicazione pubblici per l'informazione e l'educazione, continue pressione affinché definissero e ridefinissero le loro mission e le loro competenze, ma hanno sofferto come istituzioni a causa delle pressioni esercitate dal mercato e dai governi, così come da una crisi della loro immagine pubblica e, di conseguenza, della loro legittimazione agli occhi dei 
loro spettatori e ascoltatori. Per esempio, Lunt et al. hanno affermato nel loro studio sull'impatto dei dibattiti pubblici sulla BBC, che le questioni sul ruolo del pubblico servizio nella società, per come sono state poste da OFCOM, hanno indebolito le posizioni della BBC anziché sostenuto lo sviluppo futuro e la mission dell'azienda. OFCOM aveva infatti chiesto se un ambiente mediatico pienamente commercializzato fosse (o dovesse essere) il panorama mediatico preferito per il futuro, danneggiando, di conseguenza, l'immagine e la ragion d'essere della BBC.

I modi in cui è mutata la governance del pubblico servizio degli ultimi anni si possono riassumere, in modo più intenso e senza un dibattito pubblico ampio, in tre strategie cardine:

i. Un pubblico servizio (emittenti televisive e radiofoniche) precedentemente autonomo o, in alternativa, decentralizzato ha subito una riduzione delle proprie capacità amministrative ed il trasferimento dei processi decisionali verso un'amministrazione centrale.

ii. Questo processo di ricentralizzazione consolida la concezione secondo cui le regioni sono periferiche rispetto ad un fulcro centrale, sia dal punto di vista culturale che da quello politico.

iii. Ciò rende, di conseguenza, il pubblico servizio più permeabile ad un controllo politico: l'interferenza diretta da parte di un centro politico rimane uno dei due maggiori ostacoli al funzionamento di una sana organizzazione. Il secondo impedimento è rappresentato dalle difficoltà finanziarie, causate da una riduzione delle fonti di entrata del pubblico servizio o dalla modifica delle norme sui canoni, limitando le entrate pubblicitarie o riducendo i sussidi.

\section{CONCLUSIONE}

L'impatto di questi cambiamenti lo si vedrà su più livelli ed a lungo termine. La distruzione del servizio pubblico rappresenta la perdita di un investimento pubblico storico e mette a repentaglio il capitale intellettivo di queste organizzazioni. Significa altresì incertezza per la protezione del patrimonio, delle attività e dell'identità culturali in futuro. Sicuramente l'impatto di questi mutamenti renderà il pubblico servizio secondario rispetto al pubblico dibattito e alla cultura, probabilmente lo marginerà verso un punto dal quale sarà incapace di mai riprendersi e offrire un'alternativa vera ai media privati. Le conseguenze di ciò per il futuro dell'Europa e delle sue regioni sono semplicemente troppo complesse per essere esposte in questa sede. 
Nel settore privato si assiste ad una riduzione del personale e degli stipendi, nonostante non sussista alcuna esigenza economica urgente. Un effetto collaterale di questi cambiamenti è rappresentato dalla progressiva marginalizzazione, e penalizzazione, del dissenso. Ciò si nota nell'uso di giornalisti come capri espiatori sia in Spagna che in Grecia. Agnes Callamard del comitato Articolo 19 ha riassunto tale concetto utilizzando le seguenti parole:

Questi licenziamenti mandano un messaggio pericoloso agli altri giornalisti, i quali potrebbero ora astenersi dal criticare il governo temendo di subire repressioni. La sospensione di moderatori (greci) potrebbe dare adito all'autocensura da parte di altri giornalisti (Articolo 19, 2012).

Il cambio nella governance del servizio pubblico si basa su condizioni economiche e politiche eccezionali, quale l'improvviso passaggio verso un "modello di business aziendale" per ERT e "l'esigenza di creare un'emittente pubblica nuova e pulita". L'implementazione di riforme giuridiche per accompagnare tali mutamenti ha richiesto che i parlamenti nazionali fossero mantenuti al di fuori dei processi decisionali al fine di minimizzare il dibattito e gli eventuali dubbi a riguardo. Anche le ricerche da parte della stampa ed il suo coinvolgimento vengono mantenuti al minimo. Inoltre, è stata osservata la nascita di nuove istituzioni per "normalizzare" il cambio nel caso di ERT e RTVE, ove vennero creati comitati o nuove aziende per rimpiazzare quelle vecchie o per gestire il cambiamento.

Nonostante tali pressioni, il ruolo del servizio pubblico rimane quello di contribuire e facilitare la creazione di ambienti di comunicazione e spazi per un sano pubblico dibattito in vari modi. Il servizio pubblico investe le proprie risorse nel giornalismo e nei programmi di cronaca e affronta aree e tematiche che i media privati non potrebbero permettersi per ragioni economiche, o per le quali questi ultimi non dispongono delle competenze necessarie. Il servizio pubblico promuove la diversità culturale nei suoi programmi e si assume determinati rischi. Il servizio pubblico investe nell'educazione e nella formazione dei professionisti dei media e dell'arte, i quali sono presenti nell'intero panorama mediatico europeo. Infine, la società ha storicamente investito nel servizio pubblico per sviluppare un capitale intellettivo che traina gli standard qualitativi dei restanti media ad ampio beneficio della cittadinanza.

I cambiamenti normativi, argomentativi ed istituzionali vanno di pari passo con la trasformazione degli spazi pubblici che il pubblico servizio rappresenta. Le costruzioni dialettiche sui i "mali" del servizio pubblico sono parte dei dibattiti dominanti sulle crisi economiche e politiche che si 
basano sui "dilemmi", sull" urgenza" e sulla "necessità". Ci ricordano fortemente la prima ondata di deregolamentazioni alla quale l'Europa assistette all'inizio degli anni 80, quando le ragioni per lo smantellamento del welfare state e la privatizzazione di assetti pubblici consistettero nell'asserzione che gli enti pubblici sono troppo lenti per innovarsi, troppo grandi, troppo burocratici e troppo restrittivi rispetto alle scelte della clientela. Ora, di nuovo sullo sfondo di una crisi economica, gli enti pubblici vengono ritenuti troppo costosi, troppo lenti per essere riformati, non sufficientemente trasparenti o superflui. Di nuovo, il discorso sull"'efficienza" e sull" urgenza" economica di soluzioni tecnocratiche per la "modernizzazione" è rivolto ai cittadini cui è stato tolto un giornalismo indipendente e serio. Gli spazi (mediati) di dibattito aperto e di critica stanno rapidamente diminuendo. Questo secondo modello di aggressione nei confronti del servizio pubblico europeo realizza due scopi: non solo mette gli spazi digitali e gli altri spazi disponibili a disposizione degli interessi privati nella "guerra" tra emittenze, ma esercita un'effettiva censura sul diritto alla libera critica. Lo scollegamento dei cittadini dalle istituzioni consolidate, inclusi i media tradizionali (Sarikakis et al. 2013) è causato dal senso di segretezza, mancata ricettività e promesse disattese da parte di politici ed altri rappresentanti, inclusi i giornalisti (Reporter senza frontiere 2011). Il malcontento pubblico e l'inquietudine non sembrano diminuire, sicché l'esigenza di mantenere il controllo sulla sfera pubblica è talmente urgente che gli Stati e le loro élite hanno risposto con violenza.

\section{BIBLIOGRAFIA}

20 Minutos (2012), Las claves de la crisis en "El País" que ha abierto un cisma entre la cúpula directiva y la redacción, 20 minutos.es, disponibile su www.20minutos.es/n oticia/1614319/0/claves/el-pais-prisa/ere-crisis/

324 (2013), Els treballadors denuncien que TV3 $i$ Catalunya Ràdio podrien acabar desmantellats per les retallades, 324, disponibile su www.324.cat/noticia/2113215/co municacio/Els-treballadors-denuncien-que-TV3-i-Catalunya-Radio-podrien-acaba rdesmantellats-per-les-retallades

Aglietta M. (2000[1979]), Theory of capitalist regulation, Verso, New York.

ABC (2012), El congreso podrá nombrar al Presidente de RTVE por mayoría, ABC, disponibile su www.abc.es/20120420/sociedad/abci-consejo-ministros-2012042015 17.html.

Alevizopoulou M. (2013), "New Democracy Truth Team: forgers with the Prime Minister's approval", in Reports from the edge of borderline democracy, disponibile su http://borderlinereports.net/2013/04/25/new-democracytruth-team-forgers-wit h-the-prime-ministers-approval/. 
Amnesty International (2012), "Police violence in Greece - Not Just 'Isolated Incidents", EUR 25/005/2012. Disponibile su www.amnesty.org/en/documents/eur 25/005/2012/en/

Amnesty International (2014), "Greece: A law unto themselves: A culture of abuse and impunity in the Greek police”, EUR 25/005/2014. Disponibile su www.amn esty.org/en/documents/eur25/005/2014/en/

Aristotle, Nicomachean Ethics, Rackham H. (ed.). Disponibile su: http://data.perseus . org/citations/urn:cts:greekLit:tlg0086.tlg010.perseus-eng1:1098a

Article 19 (2012), Greece: Press freedom under threat by government attempts to limit criticism, disponibile su http://www.article19.org/resources.php/resource/3490/e n/greece:-press-freedom-under-threat-by-government-attempts-to-limit-criticism

Article 19 (2011a), Hungarian Media Laws Q\&A in August 2011 Country Report, disponibile su www.article19.org/data/files/medialibrary/2714/11-09-01-REPOR T-hungary.pdf

Article 19 (2011b), Hungarian Media Laws Q\&A, disponibile su www.article19.org/r esources.php/resource/2714/en/hungarian-media-laws-q\&a

Baboulias Y. (2012), “It won't just be Greek journalists who suffer from free speech crackdown", in New Statesman, 30 ottobre 2012, disponibile su www.newstates man.com/politics/2012/10/it-wont-just-be-greek-journalists-who-suffer-free-speec h-crackdown.

Banisar D. (2008), "Speaking of terror - A survey of the effects of counter-terrorism legislation on freedom of the media in Europe", disponibile su www.coe.int $/ \mathrm{t} / \mathrm{d}$ ghl/standardsetting/media/Doc/SpeakingOfTerror_en.pdf

Bardoel J. e d'Haenens L. (2008a), "Reinventing Public Service Broadcasting in Europe: prospects, promises and problems" in Media Culture Society 30(3): 337355.

Bardoel J. e d'Haenens L. (2008b), "Public Service Broadcasting in Converging Media Modalities" in Convergence: The international journal of research into New Media Technologies 14(3): 351-360

Berti E. (1978), “Ancient Greek Dialectic as Expression of Freedom of Thought and Speech", in Journal of the History of Ideas Vol. 39, No. 3 (Jul. - Sep. 1978): 347370

BBC News (2013), ERT closure: Greek parties urge change of course, disponibile su www.bbc.co.uk/news/world-europe-22871734

BOE (2012), Real Decreto-ley 15/2012, de 20 de abril, de modificación del régimen de administración de la Corporación RTVE, previsto en la Ley 17/2006, de 5 de junio, disponibile su www.boe.es/boe/dias/2012/04/21/pdfs/BOE-A-2012-5338.pdf

Branas Charles C. et al. (2015), "The impact of economic austerity and prosperity events on suicide in Greece: a 30-year interrupted time-series analysis", in BMJ Open 2015;5:1

Brouillette A. (a cura di) (2011), An Assessment of the Consistency of Hungary's Media Laws with European Practices and Norms, Center for Media and Communication Studies (CMCS) Central European University, disponibile su http:// medialaws.ceu.hu/media_authority_independence.html 
Burgen (2012), "Spanish government accused of purging critics from national radio and TV", in The Guardian, 5 August 2012, disponibile su www.theguardian. com/world/2012/aug/05/spanish-government-critics-national-tv

Chakravartty e Sarikakis (2006), Media Policy and Globalization, Palgrave Macmillan, New York.

Commissione europea (2013) Statement by the European Commission on the closure of the Hellenic Broadcasting Corporation (MEMO/13/545 12/06/2013) disponibile su http://europa.eu/rapid/press-release_MEMO-13-545_en.htm

Commissione europea (2009) Commission Staff Working Document Impact Assessment Accompanying the Communication from the Commission on the Application of State Aid Rules to Public Service Broadcasting, disponibile su http://ec.europa.eu/govern ance/ impact/ia_carried_out/docs/ia_2009/sec_2009_0893_en.pdf

Congreso Diputados (2012), Proyecto de Ley de modificación de la Ley 7/2010, de 31 de marzo, General de la Comunicación Audiovisual, para flexibilizar los modos de gestión de los servicios públicos de comunicación audiovisual autonómicos (121/000009). Presentado el 21/04/2012, calificado el 24/04/20, disponibile su www.congreso.es /public_oficiales/L10/CONG/BOCG/A/A_009-01.PDF

Congreso (2013), Diario de Sesiones de las Cortes Generales Comisiones Mixtas Año 2013 X LEGISLATURA Núm. 66 DE CONTROL PARLAMENTARIO DE LA CORPORACIÓN DE RTVE Y SUS SOCIEDADES PRESIDENCIA DEL EXCMO. SR. D. JOSÉ MARÍA BARREDA FONTES Sesión núm. 13 celebrada el miércoles, 29 de mayo de 2013 en el Palacio del Congreso de los Diputados, disponibile su. www.congreso.es/portal/page/portal/Congreso/ popupcgi?CMD=VERLST\&BA$\mathrm{SE}=$ pu10\&DOCS=1-1\&QUERY=028DSCG-10-CM-66. CODI.\%29\#(Página17)

Deutsch K. e Dunham J. (2013), Press Freedom in 2012: Middle East Volatility Amid Global Decline, Freedom House, disponibile su www.freedomhouse.org/report/fr eedom-press-2013/overview-essay

Donders K. e Pauwels C. (2008), "Service Remit of Public Broadcasting Organizations Analysis of the Commission's State Aid Approach to Digitization and the Public", in Convergence 14: 295, DOI: 10.1177/1354856508091082

Douzinas C. (2013), Philosophy and Resistance in the Crisis: Greece and the Future of Europe, Wiley.

(DWP) Department for Work and Pensions, 2015 Households Below Average Income, An analysis of the income distribution 1994/95-2013/14, Tables 4a and $4 \mathrm{~b}$.

El País (2012a), "El Ejecutivo aprueba una ley para privatizar las televisiones autonómicas", in El País, 26 aprile 2012, disponibile su http://politica.elpais.com/p olitica/2012/04/20/ actualidad/1334907236_485283.html

El País (2012b), "La crisis se lleva por delante casi 200 medios de comunicación”, in El País 13 dicembre 2012, disponibile su http://sociedad.elpais.com/sociedad/20 12/12/13/ actualidad/1355414252_725575.html

EnetEnglish.gr (2013), "European Court rules against Greece over gold mine landsale", in EnetEnglish, disponibile su www.enetenglish.gr/? i=news.en.article\&id=1 552 
Euronews (2013), "ERT close down", in Euronews 11 giugno 2013, disponibile su www.youtube.com/watch? $\mathrm{v}=\mathrm{ee} 09 \mathrm{oKuRIpo}$

Eurostat (2012), In 2011, 24\% of the population were at risk of poverty or social exclusion, Eurostat Newsrelease, Doc 171/2012, disponibile su http://epp.eurostat.ec.e uropa.eu/cache/ITY_PUBLIC/3-03122012-AP/EN/3-03122012-AP-EN.PDF

Ford M. (2014), "Europe's democratic deficit is getting worse", in The Atlantic Monthly, maggio 2014, disponibile su www.theatlantic.com/international/archive/20 14/05/europes-democratic-deficit-is-getting-worse/371297/

Freedom House (2014) Report on Impunity, disponibile su http://freedomhouse.org/ sites/default/files/Combating\%20Impunity\%20-\%20Transitional\%20Justice $\% 20$ and\%20Anti Corruption.pdf

Global Voices (2013), Italia: Se busca funcionario de prensa. 300 euros mensuales, disponibile su http://es.globalvoicesonline.org/2013/09/29/italia-se-buscafuncionar io-de-prensa-300-euros-mensuales/

Government of the Netherlands (2013), Public broadcasters receive funding from central government to provide content that is independent, varied, of high quality and accessible to a broad public, disponibile su www.government.nl/issues/media-and-br oadcasting/broadcasters

Habermas J. (1984), Theory of Communicative Action, Vol 1: Reason and the Rationalization of Society, English translation by Thomas McCarthy. Boston: Beacon Press (pubblicato originariamente in lingua tedesca nel 1981).

Held D. (2004), "Democratic Accountability and Political Effectiveness from a Cosmopolitan Perspective", in Government and Opposition. An International Journal of Comparative Politics 39(2): 364-391.

Iosifidis P. (2011), "The Public Sphere, Social Networks and Public Service Media" in Information, Communication \& Society, 14:5, 619-637 disponibile su http://dx. doi.org/10.1080/1369118X.2010.51435

Jakubobicz K. (2003) "Endgame? Contracts, Audits and the future of Public Service Broadcasting", in The Public 10 (3): 45-62

Jenson J. e Lipietz A. (1987), "Rebel Sons: the Regulation School”, in French Politics and Society, 5 (4).

Jessop B. (2011), Post-Fordism and the State, Center for Digital Discourse and Culture, disponibile su www.cddc.vt.edu/digitalfordism/fordism_materials/jessop3. htm

Kedikoglou S. (2013), Speech announcing the closure of ERT, disponibile su www.you tube. $\mathrm{com} /$ watch? $\mathrm{v}=\mathrm{h} 46 \mathrm{UwF} 9 \mathrm{tfAs}$

Lunt P. et al. (2012), "Changing regimes of regulation: implications for public service broadcasting". In Lowe G. F. and Steemers J. (ed.) Regaining the initiative for public service media, RIPE@2011. Nordicom, University of Gothernberg, Gothenburg, Sweden. Disponibile su http://eprints.lse.ac.uk/48959/1/_Libfile_r epository_Content_Lunt,\%20P_Changing\%20regimes\%20of\%20regulation $\% 281$ sero\%29.pdf 
Milanuncios (2013), Atención, periodista: ¿Harías 28 artículos al día por menos de 300 euros?, disponibile su www.clasesdeperiodismo.com/2013/08/18/atencionperiodi sta-harias-28-articulos-al-dia-por-menos-de-300-euros/

Miller L. (2013), "In Greece, Journalists Occupy the ERT Studios and Keep Producing News - Despite Austerity", in Measures Global Comment, disponibile su http://globalcomment.com/in-greece-journalists-occupy-the-ert-studios-and-keep -producing-newsdespite-austerity-measures/

Millar P. (2013), "What's all this about the EU's 'democratic deficit'?" in The Guardian, 20 May 2013, disponibile su www.theguardian.com/commentisfree/2013/ may/20/eu-democratic-deficit/print

Mithschka K. e Unterberger K. (ed.) (2013), Why Greece Matters: Public Service Media in Europe, Vienna, Österreichischer Rundfunk, ORF.

Moravcsik A. (2004), "Is there a 'Democratic Deficit' in World Politics? A Framework for Analysis", in Government and Opposition. An International Journal of Comparative Politics 39(2): 336-363.

Mosco V. (2009), The Political Economy of Communication, SAGE.

Mosco V. e McKerchner (2008), The Laboring of Communication: Will Knowledge Workers of the World Unite?, Lanham, Md: Lexington Books.

Muñiz A. (2013), El Código Penal castigará enviar tuits que inciten a alterar el orden público, disponibile su www.publico.es/470323/el-codigo-penal-castigaraenviar-t uits-que-inciten-a-alterar-el-orden-publico

Murado M. A. (2012), “Spain's cowardly purge of the journalists who ask difficult questions" in The Guardian, 7 agosto 2012, disponibile su www.theguardian.co $\mathrm{m} /$ commentisfree/2012/aug/07/spain-purge-journalists-government-votes

Nevradakis M. (2013), "Chronicling the Greek Government's Shutdown of ERT“, in Daily Kos, 17 June 2013, disponibile su www.dailykos.com/story/2013/06/17/ 1216716/-Chronicling-the-Greek-Government-s-Shutdown-of-ERT

OSCE (2013), OSCE media freedom representative says developments in Lithuania harmful to freedom of expression. Comunicato stampa disponibile su www.osce.or $\mathrm{g} /$ fom $/ 106895$

OSCE (2007), sondaggio completo disponibile su: www.osce.org/fom/24893

Parlament de Catalunya (2013), "Compareixença de Núria Amat-Sans sobre expedient de regulació d'ocupació (ERO) (tram. 357-00307/10)”, Comissió de Control de l'Actuació de la Corporació Catalana de Mitjans Audiovisuals, Diari de Sessions del Parlament de Catalunya. X leg.a S. C - N.148 2n període 28 juny 2013.

Parlamento europeo (2014), Risultati delle Elezioni europee 2014, disponibile su www.results-elections2014.eu/en/turnout.html

Pfanner E. (2012), "Portuguese Chafe as Government Examines Privatizing Broadcaster", The New York Times, 9 settembre 2012. Disponibile su www.nytimes.co $\mathrm{m} / 2012 / 09 / 10 /$ business/media/portugal-examines-privatizing-public-broadcasterrtp.html 
Proyecto LO (2013), Proyecto de Ley Orgánica por la que se modifica la Ley Orgánica 10/1995, de 23 de noviembre, del Código Penal. Presentado el 24/09/2013, calificado el 01/10/2013.disponibile su www.congreso.es/portal/page/portal/Congreso/Con greso/Iniciativas?_piref73_2148295_73_1335437_1335437.next_page=/wc/enviar CgiBuscadorIniciativas

Psychogiopoulou E. e Kandyla A. (2013), "Media policy-making in Greece: Lessons from digital terrestrial television and the restructuring of public service broadcasting”, in International Journal of Media and Culture Politics, 9.2.

Psychogiopoulou E. et al. (2011), "Does media policy promote media freedom and independence? The case of Greece”, European Commission, Brussels. Available at www.mediadem.eliamep.gr/wp-content/uploads/2012/01/Greece.pdf.

Pytlakowski Piotr (2015), Polityka No. 23 (3012), 1 to 9 June 2015

RNW (2010), disponibile su www.rnw.nl/english/bulletin/dutch-public-broadcasti ngemployees-launch-campaign-against-cuts

Reisenbichler A. e Morgan K. (2013), "How Germany Won the Euro Crisis. And Why Its Gains Could Be Fleeting", in Foreign Affairs disponibile su www.foreign affairs.com/articles/139520/alexander-reisenbichler-and-kimberly-j-morgan/how germany-won-the-euro-crisis

Reporter senza frontiere (2011), Greece. Is the crisis in Greece a chance for its media? Investigation Report, disponibile su http://en.rsf.org/IMG/pdf/rwb_greece_2011_ bd2.pdf

Reporter senza frontiere (2011-12), World Press Freedom Index 2011-2012, disponibile su http://en.rsf.org

Reporter senza frontiere (2013), World Press Freedom Index 2013, disponibile su http://en.rsf.org

Reporter senza frontiere/Indice sulla censura (2012), Riot police deliberately attack journalists covering street demonstrations, disponibile su https://www.ifex.org/greec e/2012/04/10/riot_police/

Sara Enli G. (2008), "Redefining Public Service Broadcasting Multi-Platform Participation" in Convergence: The International Journal of Research into New Media Technologies. Vol 14(1): 105-120

Sarikakis K. (2012), "Crisis - Democracy - Europe: Terms of contract? Framing public debates of the crisis" in Workshop of the Austrian Research Association on the Financial Crisis of the 21st Century, Österreischische Forschungsgemeinschaft.

Sarikakis K. (2007), "Mediating Social Cohesion: Media and Cultural Policy in the European Union and Canada" in European Studies 24: 65-90.

Sarikakis K. (2004), Powers in Media Policy, Oxford/Berna/Bruxelles/Francoforte-sulMeno/New York/Vienna, Peter Lang Academic Publishers, ISBN 3-03910-1463 and US ISBN0-8204-6956-4.

Smith F. (2012), “Greek editor Kostas Vaxevanis faces retrial over 'Lagarde list' revelation", in The Guardian, 16 novembre 2012, disponibile su www.theguardian.c om/world/2012/nov/16/greek-editor-kostas-vaxevanis-retrial 
Smith H. (2011), "Greek woes drive up suicide rate”, in The Guardian, 18 dicembre 2011, disponibile su www.theguardian.com/world/2011/dec/18/greek-woes-suici derate-highest

Sum e Jessop (2013), Beyond Regulation Approach, Edward Elgar Publishing, UK.

The Guardian (2012), "Spanish helpline reports rise in number of callers considering suicide" in The Guardian, 5 settembre 2012, disponibile su www.theguardian .com/world/2013/sep/05/spanish-helpline-rise-callers-considering-suicide

UNDP (2004), "Supporting Public Service Broadcasting - Learning from Bosnia and Herzegovina's experience”, United Nations Development Programme - Bureau for Development Policy. Disponibile su www.undp.org/content/dam/apla ws/publication/en/publications/democratic-governance/oslo-governance-center/ ogc-fellowshippapers/supporting-public-service-broadcasting-learning-from-bosn ia-andherzegovinas-experience/PublicServiceBroadcasting.pdf

Unione europea di radiodiffusione (2011), EBU Appeals to Hungarian Prime Minister to ensure media pluralism, disponibile su www3.ebu.ch/cms/en/sites/ebu/contents /news/2011/ebu-appeals-tohungarian-prime-m.html

Unione europea di radiodiffusione (2013a), EBU urges The Hague to scrap $€ 100$ million broadcasting cut, disponibile su www3.ebu.ch/cms/en/sites/ebu/contents/new s/2013/10/ebu-urges-the-hagueto-scrap-100.html

Unione europea di radiodiffusione (2013b), DG urges Romanian lawmakers to leave PSM leadership in office, disponibile su www3.ebu.ch/cms/en/sites/ebu/contents/ news/2013/02/dg-calls-on-romanianpoliticians.html

Unione europea di radiodiffusione (2013c), EBU concerned over Portuguese broadcasting budget cuts, disponibile su www3.ebu.ch/contents/news/2013/11/ebu-concer ned-over-portuguese-br.html

Unione europea di radiodiffusione (2015), EBU warns Bosnian public service broadcaster is 'close to collapse', disponibile su http://www3.ebu.ch/contents/news/2015/0 5/press-release-ebu-warns-bosnian.html

Vrousalis N. (2013a), The fascistisation of the Greek State, New Left Project, disponibile su www.newleftproject.org/index.php/site/article_comments/the_fascistisati on_of_the_greek_state

Vrousalis N. (2013b), Europe's black comedy, disponibile su www.vrousalis.net/su mmary.pdf

Zenakos A. (2013), "Reporter threatened over Aegean oil smuggling report", in Reports from the edge of borderline democracy, disponibile su http://borderlinereports. net/2013/02/03/death-threats-from-man-self-identified-as-aegean-oil-magnate/ 
